\title{
Novel Consumer-to-Product Interactions with Context-Aware Embedded Platforms
}

\author{
Sönke Knoch, Matthieu Deru, Simon Bergweiler, and Jens Haupert \\ German Research Center for Artificial Intelligence \\ Research Department Intelligent User Interfaces \\ Saarbrücken, Germany \\ \{firstname.familyname\}@dfki.de
}

\begin{abstract}
In this work we suggest a new device to instrument everyday objects in the end-user life cycle phase that makes everyday life easier for the elderly. The instrumentation of products, such as food, transforms them into smarter and more intelligent products. The equipped product notifies the user when food is spoiled and - interconnected with other smart products - advises against side effects that occur when food is consumed while a certain medication is ingested. We describe the considerations that were made towards a first prototype from a technical perspective. An interaction model was developed, a requirements analysis performed and several design and development considerations made. An architecture shows the information flow between sensors, actuators, and the Internet of Things. In the end, the prototype of a product sleeve is presented that is easy to handle and intuitive to operate. A feedback study is planned in the near future.
\end{abstract}

Keywords: Internet of Things, smart/intelligent products, prototyping.

\section{Introduction}

The proportion of elderly in the population has increased. According to the German Federal Office of Statistics [1, about one person out of seven was younger than the age of 15 years in Germany in 2010. Worldwide, only Japan had a lower rate of young people in the same year. This increasing number of old persons leads to an increasing demand in care services. New technologies can allow these growing populations of elderly to live in better conditions at home for a longer time. Sensing capabilities of old people that are fading away can be absorbed by smart devices with sensors and specific features in the smart home $[2]$ of the future. In contrast to systems which try to detect critical situations by monitoring a person's vital parameters [3] or by interpreting movement patterns [4], we seek to make life easier by instrumenting the living environment. Following the ideas of the Internet of Things [5] and Ubiquitous Computing [6], future environments will become more intelligent when a net of intelligent, smart objects is present on a larger scale.

C. Stephanidis and M. Antona (Eds.): UAHCI/HCII 2014, Part III, LNCS 8515, pp. 513-524, 2014.

(C) Springer International Publishing Switzerland 2014 
The input modalities of an intelligent, smart product collect a huge amount of data during its life cycle, so called "Big Data" [7, that can be stored in digital product memories as demonstrated by Brandherm and Kröner [8]. Contextaware computing 9 adds value to raw sensor data as it helps to understand it 10. The object memory model (OMM) as discussed in the W3C OMM Incubator Group [11] provides a format to structure data on these "smart labels". Associated data is stored on the physical artifact's label or in the digital cloud, depending on the storage capabilities of the label and the amount of data to store. A framework and a set of tools to handle OMMs is suggested by Haupert [12. It allows the creation of new applications to digital object memories. We will apply the OMM to the product sleeve, because it allows us to store information in a structured and easy to access manner and at the same time it supports the upload of code snippets [13. Code snippets define a logical rule, to perform an action (e.g., alert the user) when a certain threshold (e.g., freezing temperature) is reached.

As in many cases, objects and products today are not instrumented on grounds of costs, we suggest an intelligent product sleeve - the Ring - that makes products kinetic, tangible, smarter, and more intelligent when it is slipped on a product. While products in the future will be endued with radio frequency identification (RFID) chips [14, the product informs the sleeve smoothly about its identity and origin over near field communication (NFC). Products with barcodes are scanned with an inbuilt optical sensor or another device in the Internet of Things, e.g., a smartphone. In comparison to systems that are integrated into an object, the concept behind the product sleeve improves the cost-benefit ratio of instrumentation as it is reusable and adaptive. Therefore, we follow the concept of "Incycling" as suggested by Brandherm, Kröner, and Haupert [15].

In the following section, we will summarize related work. In Section 3 the underlying scenario for the application of the suggested prototype is described. Then, in Section 4 the instrumentation of an object, the interaction between objects and between humans and objects is modeled. In Section 5 we define the requirements based on the scenario and the interaction model from a hardware perspective. Design and software engineering decisions are depicted in the Sections [6] and 7. Finally, we summarize the results and give an outlook on future work in Section 8

\section{Related Work}

Milky [16] is an anthropomorphic milk carton that presents a new way of emotional interaction with products. The instrumented milk carton has a touch screen and sensors as input modalities and WLAN and ZigBee to communicate with other devices. The milk carton shows emotions via a face on the screen. If a customer comes closer to Milky, it blinks with its eyes and plays acoustic sounds to catch the customer's attention. As the instrumentation of such a milk carton might be too expensive, the product sleeve presented in this work depicts a cheap alternative way of product instrumentation, which is reusable and 
follows the idea of "Incycling" [15. The product sleeve was developed for home scenarios, while Milky unlocks its full potential primarily in shopping scenarios.

Mother and its Motion Cookies [17] were presented at the CES 2014 and represent one central node (Mother) with power plug and several sensor nodes (Motion Cookies) that send data to the central node. The basic package comes with one movement sensor, one thermometer, one range sensor, and one adaptive sensor node supplied by battery. These sensor nodes can be attached to objects, such as bottles, in the user's household to allow them to communicate their status to the mother following the idea of the Internet of Things. In comparison to the Mother network, the product sleeve is independent and needs no central entity as it forms the network in an ad-hoc manner.

Smart Products as described by Mülhäuser [18] can interact with the user in all life cycle phases. The product sleeve makes a product smart and is meant to be a home application. Thus, the human-to-product interaction model was developed for the end user life cycle phase. Capabilities, such as sensors, LEDs, and communication interfaces, support a natural and intuitive interaction with the equipped product. The interaction modalities are able to analyze the environment and to derive conclusions based on this knowledge. The results of this process are communicated to the user in form of guidance. A guidance is a recommendation what the user can do with the equipped product that has been in a certain environment for a while (since the moment of equipping). A minimalistic design supports natural and intuitive interaction. The product sleeve also allows the integration of the product into smart environments, such as a smart kitchen [2].

Towards smart products Meyer, Främling, and Holmström [19] suggest the term Intelligent Product. According to Gershenfeld [20], the barrier between things and the digital world has to melt in the near future and it already has looking at the last decade. The complete connection of both, the physical and the digital world remains a challenge for the future. In industrial settings, intelligent products already experienced a change through the introduction of Auto-ID technologies, where the MIT Auto-ID Center played and now the Auto-ID Labs play a crucial role. Auto-ID technologies allow products to communicate their current state to the machines that handle the product. For example, Kröner et al. 21] demonstrate the interaction with digital product memories by showing how a robot handles fragile and non-fragile objects in different ways, throwing them into the basket or placing them carefully on the table. All these changes and new opportunities resulting from the rise of new technologies trigger a shift from centralized to distributed computing in production. These changes may end up in the Fourth Industrial Revolution, Industry 4.0 [22], a research strategy exclaimed by the German Federal Ministry of Education and Research. In this context, the Internet of Things, Cloud Computing, and Big Data are the most influential factors.

According to the classification for intelligent products by Meyer et al. [19], the suggested product sleeve has a level of intelligence that is called problem notification. Because of its ability to detect problems and communicate them to 
the user its intelligence is above simple information handling. Still, it has not reached the level of decision making which is up to the user. Its nature could be better described as a decision support system. The location of intelligence is an intelligence at object and partly an intelligence through network. On the one hand, the object instrumented with the sleeve has all computational power, storing capacities, and connectivity that is necessary and, thus can be called a big smart device or embedded platform. On the other hand, knowledge about objects in the vicinity may influence notifications and decision support. Finally, regarding the aggregation level of intelligence the product sleeve is an intelligent container, as it is aware of an object that might be intelligent by itself. It registers if an object is removed or attached, adapts and processes the object's knowledge, and is not an intelligent item that only manages information and notifications about itself.

\section{Scenario}

John is an old person who has difficulties to manage the challenges of everyday life. He suffers from mild cognitive impairments and forgetfulness. Nevertheless, John would rather not move to a retirement home. A care service supports him and passes by every day to undertake a few basic tasks, e.g., shopping, cleaning, and looking after the patient's condition. The time for these tasks is limited, such that the service only has half an hour for each visit. In the time left, John is on his own. Because his senses are fading away while getting older, it has become more difficult for him, e.g., to deal with the groceries in the household. The information on the packages is printed in very small letters and is hard to read. It has also become harder for John to smell or see if a product is spoiled. The product sleeve that is attached to food by the employee of the care service when she brings the groceries and places them into the fridge, visualizes product information in such a way that John can recognize it easier and detects spoiled food by the product's date of expiry. As the product sleeve follows the idea of "Incycling", it can be reused on similar products in the future. An inbuilt accelerometer recognizes movement when the product is grabbed by John and alerts him if the product has expired. A red light and an acoustic alert hint at the potentially spoiled food. If the food is okay and the storage of the food was all right - appropriate temperature and brightness - then the light shines green.

\section{Interaction Model}

\subsection{Object Instrumentation}

The sleeve device is charged in a charging cradle and can be applied to a variety of products. Our prototype was made to carry bottles and other drinking vessels, such as beverage cartons commonly utilized to carry milk or juice. To instrument the object with the sleeve device, the RFID interface has to be placed very close to the product's RFID chip to allow the device to read the data on the chip. If no 
RFID chip is available at the product, the barcode is scanned with the camera of the device or of a smartphone. Once the data is transmitted, the device can fetch additional information over its Wi-Fi connection. Related snippets are downloaded to build the basis for notifications on status changes of the product. Now, the newly instrumented product has become a smart, intelligent product and can be used as usual with its additional, newly won features.

\subsection{Human-to-Object Interaction}

Human-to-object interaction is triggered in two ways indirectly and directly. An indirect interaction is given when the sensors detect that the user is paying attention to the object. Movement detection, brightness, and distance measurements register when the object is grabbed. If a notification is waiting in the queue to get communicated, it is now released. The output modality depends on the priority of the notification. High priority notifications are released automatically. The object vibrates, shows red light over the LED, and plays the message over the audio speaker. An example for such a high priority notification would be that the milk has expired. Low priority notifications are visualized over an orange light. If the user wants to hear the notification he or she can push the button to hear the notification. An example for a low priority notification would be that the milk is almost finished. If there are no notifications in the queue the LED shows green light. To rate the notification, the user has to press the one touch button interface that provides a feedback function.

\subsection{Object-to-Object Interaction}

Multiple instrumented objects that use the OMM to store data can also interact with each other. Over Web service discovery the objects know about the existence of each other and can generate notifications based on code snippets [13] they have automatically downloaded. One example is the suggestion of receipts. For example, the milk can suggest to make pancakes if it registered a carton of eggs in the same fridge. This would be a low priority notification. An example for high priority notifications are side effects that occur when a certain kind of food is consumed while the patient is ingesting a certain medication. For instance, drinking grapefruit juice can increase the level of medicine in the blood, which can increase the risk of side effects. Calcium channel blockers, a medication to decrease blood pressure in patients with hypertension, can interact with grapefruit juice and increase the level of medicine in the patient's blood. The system prevents the consumption of both and requests the user to seek advice from the pharmacist or doctor. As the product sleeve in its current, prototypic state is not suitable to be attached to pills, the interconnected object would be an intelligent medicament blister, as suggested by Nesselrath et al. 2].

\section{Requirements}

To support the interaction model presented in Section 4 a set of requirements for the device can be derived. 


\subsection{Input Modalities}

An accelerometer is necessary to detect movements of the device. A distance measurement module allows to detect if a person or object is close to the Ring. A light sensor will detect the brightness around the object. This function is necessary to check for example, if the fridge is open or not and to monitor the storage conditions. To supervise storage conditions, a temperature and humidity sensor will provide an additional sense.

\subsection{Output Modalities}

To answer the question about the visualization, it is important to discuss the target group of users and the planned shape of the product. The users will be elderly who experience a fading away of their senses through normal aging up to mild cognitive impairments. An easy-to-use and self-explaining user interface has to be developed to support such users. Touchscreens might provide a lot of capabilities, but are difficult to fit in a round shaped device that fits around a bottle. For that reason, we will use LEDs to visualize the state of the product (in good condition, in bad condition, there might be an issue) that illuminate the product sleeve in green, red, or orange light. To communicate the content of the notification to the user, an easy to recognize output modality has to be chosen. As displays are difficult to integrate and hard to read, especially for the elderly, natural language output in form of spoken words seems to be most appropriate. From a hardware point of view, this requires the integration of a speaker. For people suffering of deafness, the visualization is submitted to a screen device in the vicinity, e.g., the television or the tablet. If not understood at the first time, the playback of the natural reader can be played back again by pressing the button.

\subsection{Communication}

A camera is on-board to provide an interface to read visual tags, such as barcodes. NFC allows to read and write information that is stored on RFID chips. Over Wi-Fi the connection to the Internet can be established to fetch additional information that might be necessary to generate product related notifications. ZigBee is a communication protocol that allows wireless communication between devices consuming low energy. To allow communication between objects in the vicinity in an energy saving way, a ZigBee module is integrated.

\subsection{Power Supply}

To run the required functionality, an appropriate power supply is necessary. The intended hardware to realize all input and output modalities is consuming little power. Main consuming components are the CPU and the communication module. So it is an advantage if some of the main consumers known from the smartphone domain (cf. [23, [24]), such as LCD and GPS, are not inbuilt. To 


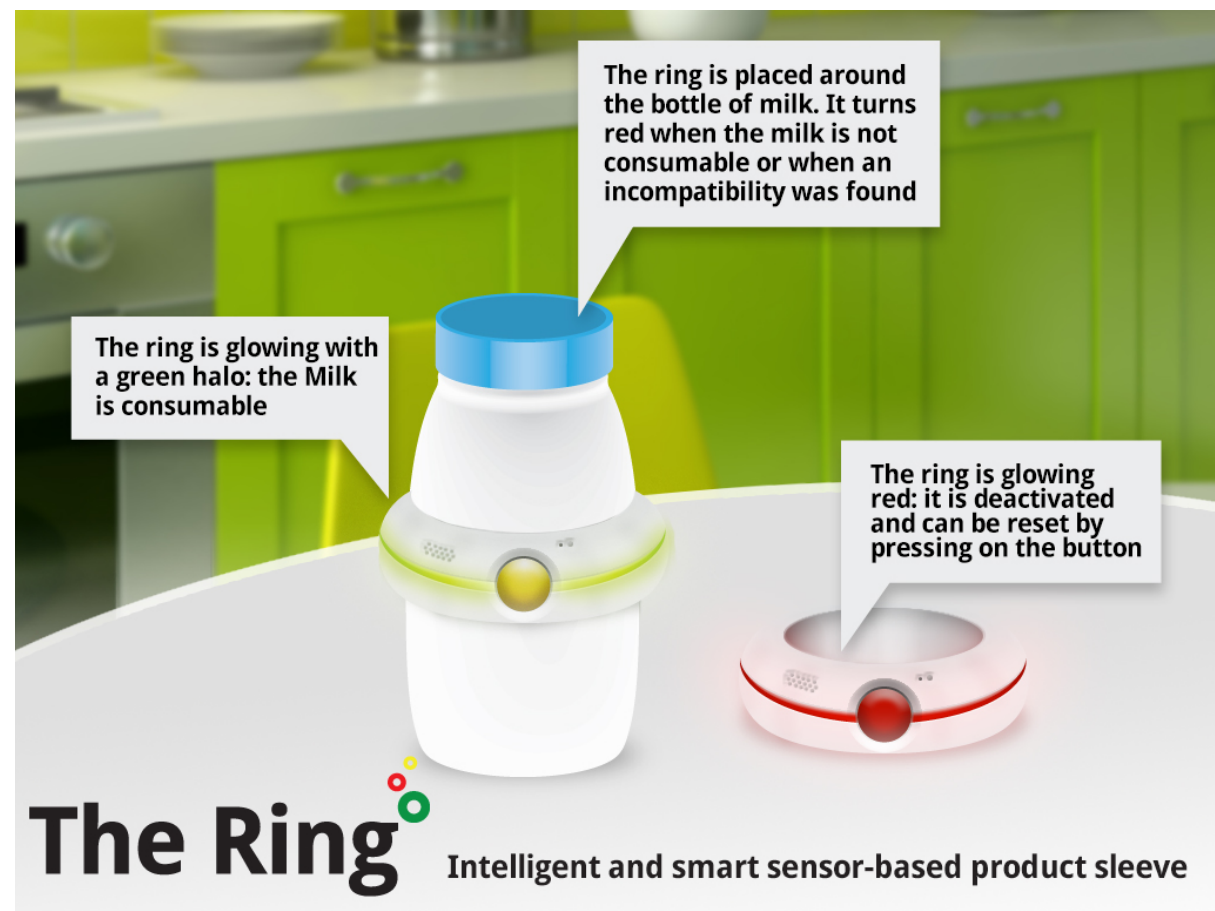

Fig. 1. Shape of the Ring

load the mobile power supply, the product sleeve is placed in a charging cradle that loads the device wireless. This way of charging is easy to handle for the care service and the elderly, as no cable has to be plugged in and out.

\section{Design}

The design of the Ring follows the philosophy of creating a minimalistic device. The object instrumentation as described in Section 4.1 has to be very easy. Additionally, there should be only one intuitive "interface" between device and user. We realize this idea with a one button interaction. The button is integrated in an elastic band. It changes its color according to the tracked sensor information, which trigger low or high priority notifications. When, for example, the milk is in good condition and no compatibility problems with medicines or the metabolism of the consumer were found, it will turn green. On the other hand, if there is a potential problem with the milk, the button will turn red. When recommendations and suggestions - low priority notifications - are generated the button will turn orange. In order to amplify this visual signal and to provide a direct, clearly visible feedback to the user, a small flexible LED stripe is placed around the elastic band. This stripe will glow accordingly red, green, or orange. These colors are amplified by sequences of light impulse. The batteries and the sensors 
are placed in the elastic band making it completely autonomous. Figure 1 shows the design concept of the Ring.

The one button concept plays a central role. It allows the caring personnel to easily reset the status of the ring by pressing the central button for 10 seconds. This action resynchronizes the parameters and the sensors, for example, when the ring was placed on a new bottle of milk. To parametrize the ring, wireless communication is used: over an app and a Wi-Fi module, the caring personnel can set up several parameters depending on the patient's health or metabolism. This configuration defines when the ring has to glow and what parameters of the milk are taken into account over the integrated sensors.

The consumption of the patient is also registered by the Ring: each time the patient takes the bottle and opens it, a log entry is written into the storage. This information is used to alert the caring personnel if the patient has not correctly followed the medical advices and "consumed" the product as advised. This kind of behavior tracking can be useful and vital especially for elderly patients. For example, during the summer, the Ring could be used to check if a person has drunk enough water. This prevents a feeling of weakness and the risk of dehydration during heat waves.

\section{Technical Infrastructure}

To develop a first prototype of the product sleeve that fits the requirements described in Section 5, we decided to use the Microsoft .NET Gadgeteer platform. Microsoft .NET Gadgeteer provides a platform that is easy to learn and represents a good basis for quick prototyping networked devices for the Internet of Things 25].

The core component of the prototype that was developed in this approach based on a GHI Electronics Spider Board running the .NET Micro Framework. There are different types of sensors that can be connected to this Gadgeteer platform. The sensor interpretation layer that can be seen in Figure 2 gathers information of the current environment to set situational parameters. These parameters are necessary in order to make an evaluative statement and establish the basis for estimations and forecasts. In the implemented prototype, we use a moisture and temperature/barometer sensor combined with an accelerometer and gyroscope module. A barometer module analyzes the current weather situation and will check if, for instance, milk is beginning to turn sour in the following hours. Additionally, a mobile accumulator provides the power supply for the modules and the Gadgeteer board.

On the software side, the framework for estimation and forecasting based on sensor knowledge is written in $\mathrm{C \#}$, the standard programming language for the .NET framework. The evaluation and case analysis will be based on defined rules. Once a rule matches, an action is triggered and sent to the Presentation Manager. This module initiates the appropriate form of visualization, supported by pre-defined acoustic alerts. Not every use case allows the use of a touchscreen, but a touchscreen has the advantage that information can be displayed directly. 

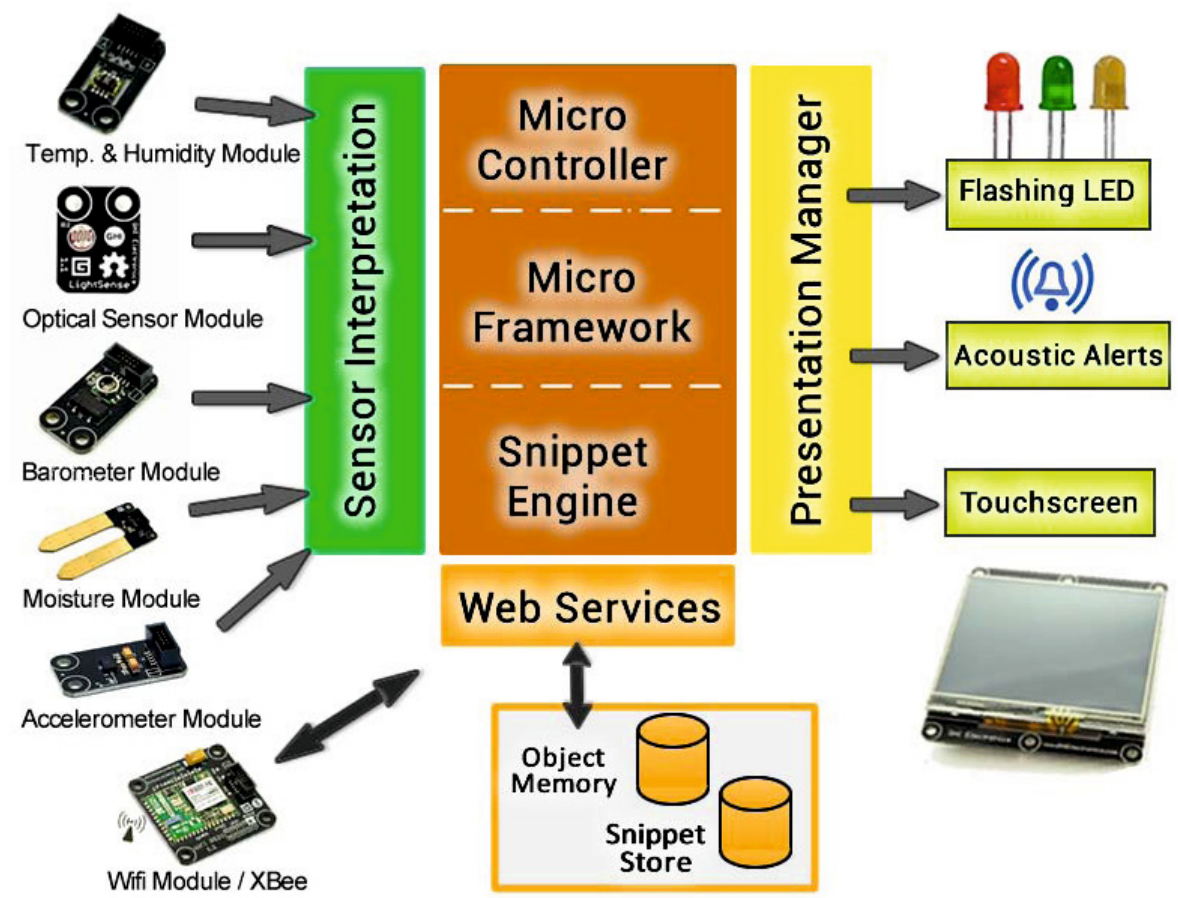

Fig. 2. Technical Architecture and a Set of Selected Sensors

It encourages the user to interact directly via gesture. In this way, for example, a conscious acknowledgement is obtained and given that a warning has been read. In its minimal lightweight variant that can be seen in Figure 1, the system can also be assembled just with LEDs that show the relevant states and illuminate the Ring. In this case, the status varies from red to green, from inedible to edible. A network layer enables the access to Web services over a Wi-Fi connection. Conditions and information can be sent to the server that hosts all object memories and receives updates from the snippet store.

\section{Conclusion and Future Work}

In this work, we presented the Ring that instruments everyday objects and transforms them into smart, intelligent objects. Its context-awareness allows the communication of notifications about the state of the attached object to the user. The realization showed that the system supports both, the interaction between humans and objects as well as between interconnected objects, follwing the idea of the Internet of Things. As an application, we suggested the household of an elderly person. The Ring can be used to reinforce the person's decreasing senses. 
It alerts the user about the exceeding of expiry dates or about side effects with certain medications. An interaction model supports these scenarios. A technical architecture regulates the information flow within the device, where the main parts are the Sensor Interpretation, the Web service, and the Presentation Manager. The Web service provides a communication interface to data sources and to other objects in range. The Object Memory Model (OMM) is used to structure information in the object's storage, in the device or in the cloud, depending on the amount of data.

We are building a good-looking prototype of the presented device using the Gadgeteer platform and a 3D printer. To receive some feedback about the device's utility, it is planned to consult a group of elderly people about the operational fitness in everyday life in the near future. Additionally, the application of the device in different scenarios is planned. One example is the instrumentation of expensive products, such as champagne or wine bottles, in a shopping environment. It is also planned to extend the suggested approach to objects with different shapes and sizes. This would apply the concept of "Incycling" to a wider range of products reducing hardware costs and the environmental impact in future shopping scenarios by reusing the hardware.

Acknowledgment. This research was funded in part by the German Federal Ministry of Education and Research under grant number 01IA11001 (project RES-COM) and 01IS13015 (project SmartF-IT). The responsibility for this publication lies with the authors.

\section{References}

1. Statistisches Bundesamt (Destatis): Just released - statistical yearbook (October 2012), www.destatis.de/EN/PressServices/Press/pr/2012/10/PE12_351_p001.html (accessed at January 29, 2014)

2. Nesselrath, R., Haupert, J., Frey, J., Brandherm, B.: Supporting persons with special needs in their daily life in a smart home. In: 2011 7th International Conference on Intelligent Environments (IE), pp. 370-373 (2011)

3. P, G.: HOPE- eectronic gadget for home bound patients and elders: Create the future design contest (June 2012),

\section{http://contest.techbriefs.com/2012/entries/medical/2659}

(accessed at January 29, 2014)

4. Dovgan, E., Lutrek, M., Pogorelc, B., Gradiek, A., Bruger, H., Gams, M.: Intelligent elderly-care prototype for fall and disease detection. Slovenian Medical Journal 80(11) (January 2011)

5. Ashton, K.: That 'internet of things' thing (June 2009), Ww.rfidjournal.com/articles/view?4986 (accessed at January 29, 2014)

6. Weiser, M.: Some computer science issues in ubiquitous computing. Commun. ACM 36(7), 75-84 (1993) 
7. Zaslavsky, A., Perera, C., Georgakopoulos, D.: Sensing as a service and big data. arXiv:1301.0159 [cs] (January 2013), Proceedings of the International Conference on Advances in Cloud Computing (ACC), Bangalore, India (July 2012)

8. Brandherm, B., Kröner, A.: Digital product memories and product life cycle. In: 2011 7th International Conference on Intelligent Environments (IE), pp. 374-377 (2011)

9. Dey, A.K.: Understanding and using context. Personal Ubiquitous Comput. 5(1), 4-7 (2001)

10. Perera, C., Zaslavsky, A.B., Christen, P., Georgakopoulos, D.: Context aware computing for the internet of things: A survey. IEEE Communications Surveys \& Tutorials Journal abs/1305.0982 (2013)

11. Kröner, A., Haupert, J., Seiler, M., Kiesel, B., Schennerlein, B., Horn, S., Schreiber, D., Barthel, R.: Object memory modeling (September 2011),

www.w3.org/2005/Incubator/omm/XGR-omm-20111026/ (accessed at January 29, 2014)

12. Haupert, J.: Domeman: A framework for representation, management, and utilization of digital object memories. In: Augusto, J.C., Bourdakis, V., Braga, D., Egerton, S., Fujinami, K., Hunter, G., Kawsar, F., Lotfi, A., Preuveneers, D., Binabdulrahman, A.W., Zamudio, V. (eds.) 9th International Conference on Intelligent Environments (IE 2013), July 18-19, pp. 84-91. IEEE, Athen (2013)

13. Kröner, A., Haupert, J., Hauck, C., Deru, M., Bergweiler, S.: Fostering access to data collections in the internet of things. In: Narzt, W., Gordon-Ross, A. (eds.) UBICOMM 2013, The Seventh International Conference on Mobile Ubiquitous Computing, Systems, Services and Technologies, pp. 65-68. IARIA (September 2013) (Best Paper Award)

14. Resatsch, F., Sandner, U., Leimeister, J.M., Krcmar, H.: Do point of sale RFIDBased information services make a difference? analyzing consumer perceptions for designing smart product information services in retail business. Electronic Markets 18(3), 216-231 (2008)

15. Brandherm, B., Kröner, A., Haupert, J.: Incycling: Sustainable concept for instrumenting everyday commodities. In: Proceedings of the 2011 International Workshop on Networking and Object Memories for the Internet of Things, NoME-IoT 2011, pp. 27-28. ACM, New York (2011)

16. Deru, M., Bergweiler, S.: Milky: On-product app for emotional product to human interactions. In: Proceedings of the 15th International Conference on Human-Computer Interaction with Mobile Devices and Services, MobileHCI 2013, pp. 552-557. ACM, New York (2013)

17. Sen.se: Mother (January 2014), http://sen.se/store/mother (accessed at January 29, 2014)

18. Mühlhäuser, M.: Smart products: An introduction. In: Mühlhäuser, M., Ferscha, A., Aitenbichler, E. (eds.) Constructing Ambient Intelligence. CCIS, vol. 11, pp. 158-164. Springer, Heidelberg (2008)

19. Meyer, G.G., Främling, K., Holmström, J.: Intelligent products: A survey. Computers in Industry 60(3), 137-148 (2009)

20. Gershenfeld, N.A.: When things start to think. Henry Holt, New York (1999)

21. Kröner, A., Haupert, J., GeaFernndez, J., Steffen, R., Kleegrewe, C., Schneider, M.: Supporting interaction with digital product memories. In: Wahlster, W. (ed.) SemProM. Cognitive Technologies, pp. 223-242. Springer, Heidelberg (2013) 
22. German Federal Ministry of Education and Research and German Federal Ministry of Economics and Technology: Project of the future: Industry 4.0 (March 2013), www.bmbf.de/en/19955.php (accessed at January 29, 2014)

23. Carroll, A., Heiser, G.: An analysis of power consumption in a smartphone. In: Proceedings of the 2010 USENIX Conference on USENIX Annual Technical Conference, USENIXATC 2010, p. 21. USENIX Association, Berkeley (2010)

24. Niskanen, I., Kantorovitch, J.: Towards the future smart products systems design. In: 2011 IEEE International Conference on Pervasive Computing and Communications Workshops (PERCOM Workshops), pp. 313-315 (2011)

25. Hodges, S., Taylor, S., Villar, N., Scott, J., Bial, D., Fischer, P.T.: Prototyping connected devices for the internet of things. Computer 46(2), 26-34 (2013) 